\title{
ANALYSIS OF DIFFERENT TYPES OF THREATS ON NETWORKS AND VARIOUS MEANS OF SECURING NETWORKS
}

\author{
Manisha Wadhwa \\ India
}

\section{ABSTRACT}

This paper describes about the security that can be provided to a network from unauthorized access and risks from all the possible attacks and threats. This paper also covers preventive measures to protect networks from potential security threats.

Key words: Access Control, Data loss, Intrusion and Firewalls

\section{INTRODUCTION}

Network security consists of the policies and practices adopted to prevent and monitor unauthorized access, misuse, modification, or denial of a computer network and networkaccessible resources. Network security involves the authorization of access to data in a network, which is controlled by the network administrator. Users choose or are assigned an ID and password or other authenticating information that allows them access to information and programs within their authority. Network security covers a variety of computer networks, both public and private, that are used in everyday jobs; conducting transactions and communications among businesses, government agencies and individuals. Networks can be private, such as within a company, and others which might be open to public access. Network security is involved in organizations, enterprises, and other types of institutions. It does as its title explains: It secures the network, as well as protecting and overseeing operations being done. The most common and simple way of protecting a network resource is by assigning it a unique name and a corresponding password.Network security is the security provided to a network from unauthorized access and risks. It is the duty of network administrators to adopt preventive measures to protect their networks from potential security threats. Computer networks that are involved in regular transactions and communication within the government, individuals, or business require security. The most common and simple way of protecting a network resource is by assigning it a unique name and a corresponding password.Network security is any activity designed to protect the usability and integrity of your network and data. It includes both hardware and software technologies. Effective network security manages access to the network. It targets a variety of threats and stops them from entering or spreading on your network.

\section{PRIMARY TYPES OF NETWORK THREATS}

$>$ Structured threats

$>$ Internal threats

$>$ External threats

$>$ Unstructured threats 
DOI: https://dx.doi.org/10.26808/rs.ca.i7v6.08

International Journal of Computer Application (2250-1797)

Volume 7- No.6, November-December 2017

\section{STRUCTURED THREATS}

Structured threats are more focused by one or more individuals with higher-level skills actively working to compromise a system. The targeted system could have been detected through some random search process, or it might have been selected specifically. The attackers are typically knowledgeable about network designs, security, access procedures, and hacking tools, and they have the ability to create scripts or applications to further their objectives. Structured attacks are more likely to be motivated by something other than curiosity or showing off to one's peers. Greed, politics, racism (or any intolerance), or law enforcement (ironic) could all be motives behind the efforts. Crimes of all types where the payoff isn't directly tied to the attack, such as identity theft or credit card information theft, are also motivations. International terrorism and government-sponsored attacks on another country's computer infrastructure are becoming well documented. Systems of interest might include utilities, public safety, transportation systems, financial systems, or defense systems, which are all managed by large data systems, each with vulnerabilities.

\section{INTERNAL THREATS}

Internal threats originate from individuals who have or have had authorized access to the network. This could be a disgruntled employee, an opportunistic employee, or an unhappy past employee whose access is still active. In the case of a past network employee, even if their account is gone, they could be using a compromised account or one they set up before leaving for just this purpose. Many surveys and studies show that internal attacks can be significant in both the number and the size of any losses. If dishonest employees steal inventory or petty cash, or set up elaborate paper-invoicing schemes, why wouldn't they learn to use the computer systems to further their ambitions? With access to the right systems, a trusted employee can devastate an unsuspecting organization. All too often, employers fail to prosecute this type of activity. The reasons range from fear of the activity becoming public knowledge to knowing that, quite often, record-keeping systems haven't been developed either to provide adequate evidence or to prove that the transactions, no matter how ludicrous, weren't authorized.

\section{EXTERNAL THREATS}

External threats are threats from individuals outside the organization, often using the Internet or dial-up access. These attackers don't have authorized access to the systems. In trying to categorize a specific threat, the result could possibly be a combination of two or more threats. The attack might be structured from an external source, but a serious crime might have one or more compromised employees on the inside actively furthering the endeavor.

\section{UNSTRUCTURED THREATS}

Unstructured threats often involve unfocused assaults on one or more network systems, often by individuals with limited or developing skills. The systems being attacked and infected are probably unknown to the perpetrator. These attacks are often the result of people with limited integrity and too much time on their hands. Malicious intent might or might not exist, but there is always indifference to the resulting damage caused to others. The Internet has many sites where the curious can select program codes, such as a virus, worm, or Trojan horse, often with instructions that can be modified or redistributed as is. In all cases, these items are small 
DOI: https://dx.doi.org/10.26808/rs.ca.i7v6.08 International Journal of Computer Application (2250-1797)

Volume 7- No.6, November-December 2017

programs written by a human being. They aren't alive and they can't evolve spontaneously from nothing. The person launching an unstructured attack is often referred to as a script kiddy because that person often lacks the skills to develop the threat themselves, but can pass it on anonymously (they think) and gain some perverse sense of satisfaction from the result. E-mail delivery methods have replaced "shared" game disks as the vehicle of choice for distributing this type of attack.The term "script kiddy" is a common derogatory term and should be used with caution, if at all. Script kiddy is included here so you know what it means. Remember, the difference between an unstructured attack and a series of all-out denial-of-service attacks might be that the latter attacker is offended or angry. Unstructured attacks involving code that reproduces itself and mails a copy to everyone in the person's e-mail address book can easily circle the globe in a few hours, causing problems for networks and individuals all over the world. While the original intent might have been more thoughtless than malicious, the result can be a loss of user access while systems are being protected, a loss of reputation if the news that a company's site has been attacked, or a loss of user freedoms as more-restrictive policies and practices are implemented to defend against additional attacks. In some organizations, if the network is down, entire groups of people can't do their jobs, so they're either sent home or they sit and wait without pay because their income is tied to sales. So even if the hacker "thought" no one would be hurt, the result is often that they just beat some single parent or new hire out of a day's pay. Each of these results can be quantified in currency and often result in large numbers if and when the perpetrator is prosecuted.

\section{TYPES OF NETWORK SECURITY}

\section{NETWORK ACCESS CONTROL (NAC)}

This is when you control who can and can't access your network. You do this by identifyingwhich devices and users are allowed into your network. From there, you can enforce various security policies such as blocking certain devices and controlling what someone can do within your network. You can also utilize behavioral analytictools to identify what normal and abnormal behavior is. Once you do that, you can set it up where you'll get notifications whenever something is acting abnormally. Similarly, you can implement firewalls, which is when you put a barrier between your internal network and untrusted outside networks, such as the internet. This way, you can also control your staff's web use and block any threats or dangerous websites.

\section{APPLICATION SECURITY}

Application security is exactly how it sounds - security that protects your applications. This type of security is important to have because no app is created perfectly. They

can have a lot of holes or weaknesses where a hacker can enter. A lot of your business operations and devices may run on applications, so this type of security is a must-have.

\section{ANTIVIRUS AND ANTIMALWARE SOFTWARE}

This software is used to protect against malware, which includes anything from viruses, Trojans, ransomware, or spyware. Besides the obvious reasons, malware can be very dangerous because sometimes, it can will stay calm within your network for days and weeks, just sitting there ready to spring up and attack. Antivirus and antimalware software deal with this threat by scanning for malware entry and tracking files afterward to find any that may have slipped in and are laying low. 
DOI: https://dx.doi.org/10.26808/rs.ca.i7v6.08

International Journal of Computer Application (2250-1797)

Volume 7- No.6, November-December 2017

\section{EMAIL SECURITY}

Here's a big one. Your email is pretty important for your business, and considering that email gateways are the number one threat for a security breach, email security is an absolute vital one to have. Attackers can use your personal information to do all kinds of damage, such as blackmail or emailing on your behalf to deceive your clients and send them to sites full of malware. An email security application can help block these attacks and control what is sent out.

\section{WIRELESS SECURITY}

Here's another big one. The mobile office movement is gaining momentum, and with that comes wireless networks and access points. However, wireless networks are not as secure as wired ones, allowing more room for hacker entry, so the power of wireless security needs to be strong.Since there are many parts to your infrastructure, there are many types of security out there to protect it. There are a lot more that we didn't mention in this blog, but we know all about them here at Alliance Technology Partners.

\section{CONCLUSION}

Network security starts with Authentication, commonly with a username and a password. Since this requires just one detail authenticating the user name-i.e., the password - this is sometimes termed one-factor authentication. With two-factor authentication, something the user 'has' is also used (e.g., a security tokenor 'dongle', an ATM card, or a mobile phone); and with three-factor authentication, something the user 'is' is also used (e.g., a fingerprint or retinal scan).Once authenticated, a firewall enforces access policies such as what services are allowed to be accessed by the network users. Though effective to prevent unauthorized access, this component may fail to check potentially harmful content such as computer worms or Trojans being transmitted over the network. Anti-virus software or an intrusion prevention system (IPS) help detect and inhibit the action of such malware. An anomaly-based intrusion detection system may also monitor the network like wireshark traffic and may be logged for audit purposes and for later high-level analysis. Newer systems combining unsupervised machine learning with full network traffic analysis can detect active network attackers from malicious insiders or targeted external attackers that have compromised a user machine or account. Communication between two hosts using a network may be encrypted to maintain privacy.

\section{REFERENCES}

1. https://en.wikipedia.org/wiki/Network_security

2. A Role-Based Trusted Network Provides Pervasive Security and Compliance - interview with JayshreeUllal, senior VP of Cisco

3. Jump up^ Dave Dittrich, Network monitoring/Intrusion Detection Systems (IDS), University of Washington.

4. Jump up^ "Dark Reading: Automating Breach Detection For The Way Security Professionals Think". October 1, 2015. 